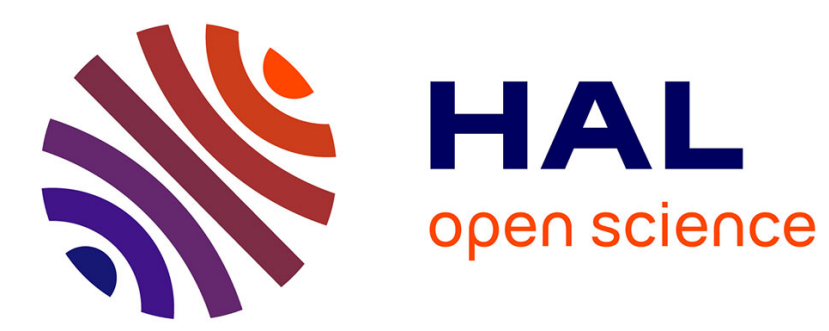

\title{
Les avatars de la pensée magique
}

Paulo Ceccarelli, Cristina Lindenmeyer

\section{To cite this version:}

Paulo Ceccarelli, Cristina Lindenmeyer. Les avatars de la pensée magique. Cliniques méditerranéennes, 2012, La pensée magique 1 (85), pp.41 - 49. 10.3917/cm.085.0041 . hal-01515537

\section{HAL Id: hal-01515537 \\ https://hal.science/hal-01515537}

Submitted on 25 Aug 2017

HAL is a multi-disciplinary open access archive for the deposit and dissemination of scientific research documents, whether they are published or not. The documents may come from teaching and research institutions in France or abroad, or from public or private research centers.
L'archive ouverte pluridisciplinaire HAL, est destinée au dépôt et à la diffusion de documents scientifiques de niveau recherche, publiés ou non, émanant des établissements d'enseignement et de recherche français ou étrangers, des laboratoires publics ou privés. 


\section{Paulo Ceccarelli \\ Cristina Lindenmeyer}

\section{Les avatars de la pensée magique}

«La comparaison avec la psychanalyse nous a permis d'éclairer certains aspects de la cure chamanique. Il n'est pas certain qu'inversement, l'étude chamanique ne soit pas appelée un jour, à élucider des points restés obscurs de la théorie de Freud. Nous pensons particulièrement à la notion de mythe et à la notion de l'inconscient. »

Lévi-Strauss

Ce texte propose quelques réflexions entre psychanalystes et anthropologues sur ce que nous pouvons appeler la pensée magique, dans certaines constructions mythiques des populations indiennes du nord du Brésil.

Comment est possible ce rapprochement entre deux mouvements de pensées à la fois opposé et si proches?

On utilise l'expression pensée magique pour désigner la croyance selon laquelle certaines pensées permettraient l'accomplissement des désirs, et aussi l'empêchement d'événements problématiques ou désagréables. Chez l'adulte, la persistance de ce type de pensée, typique de la période infantile, dénoterait un symptôme d'immaturité, voire un déséquilibre psychologique.

Cela dit, ce « reliquat de la mentalité primitive ${ }^{1}$ », subsiste toujours sous une forme latente dans le psychisme de tout un chacun. La pensée magique constitue une tentative d'échapper aux angoisses et aux conflits, du monde externe et du monde interne. Comme si l'acte de penser pouvait contrôler,

Paulo Ceccarelli, psychanalyste, professeur à l'université PUC de Minas Gerais, Brésil ; Rua Aimorés 1239/702.30140-071, Belo Horizonte, MG, Brésil - paulorcbh@mac.com

Cristina Lindenmeyer, psychanalyste, maître de conférences à l'université Paris 7 (Denis Diderot) ; 44, rue de Sévigné, F-75003 Paris - cristina.lindenmeyer@wanadoo.fr

1. L. Lévy-Bruhl (1922), La mentalité primitive, Paris, PUF, 15e édition, 1960. 
expliquer et même changer la réalité. Aussi, donne-t-elle l'impression d'établir un lien causal entre deux événements indépendants. La pensée magique va au-delà des mots pour rejoindre des pouvoirs que l'on attribue, par exemple, aux cristaux supposés contenir le pouvoir de concentrer de l'énergie ; des cristaux colorés aux effets thérapeutiques particuliers ; des couleurs associées à des parties du corps; et ainsi de suite. Cette croyance très ancienne de pouvoir modifier le monde par la pensée ou par la parole n'est pas qu'une attitude propre aux enfants, mais aussi aux primitifs et à certaines névroses. On la retrouve dans toutes les civilisations - les chants sacrés de la pluie, par exemple. Elle est aussi reprise dans la Bible puisque Dieu pour créer le monde se fait «Verbe » : «Que la lumière soit et la lumière fut. »

Les histoires des peuples indiens de l'Amazonie sont très riches en exemples de pensées magiques. Maués ${ }^{2}$ et Mindlin $^{3}$ décrivent de nombreuses façons dont la pensée magique est présente dans les communautés indiennes et l'importance que ces pensées ont dans la vie quotidienne de ces sujets, en particulier sur les causes et les origines des maladies. Par exemple, Maués ${ }^{4}$ raconte des récits sur la médecine dans un village de pêcheurs - Itapuã - situé dans la municipalité de Vigia dans l'État du Pará, en Amazonie brésilienne. Pour ces sujets-là, il y a une grande différence entre les maladies naturelles et les non naturelles. Ces dernières seraient causées par divers facteurs qui peuvent être humains ou non humains, comme la sorcellerie, le mauvais œil, la force des esprits, et ainsi de suite. Maués nous raconte un événement concernant une adolescente de 17 ans qui vivait avec sa mère, veuve, et ses deux frères. La mère de la jeune fille commença à remarquer que sa fille était étrange, pâle, et que sans doute elle cachait quelque chose. La mère chercha le chaman du village qui, après une longue conversation avec la jeune fille malade, dit à la mère qu'elle prenne bien soin de sa fille parce qu'un boto ${ }^{5}$ enchanté était tombé amoureux d'elle. Pour mettre fin à cette situation, le chaman dit aux frères de la jeune fille de prendre des armes et d'attendre le boto au bord de la rivière. Selon l'auteur, après un certain temps, ils ont vu un mouvement dans l'eau, mais aucun bateau n'y était. De l'eau sortit, alors, un beau garçon habillé en blanc. Les frères n'ont pas hésité et ont tiré sur lui ; et il est tombé dans l'eau. Le lendemain, le cadavre d'un boto a été retrouvé par des pêcheurs.

2. R. H. Maués, A ilha encantada : medicina e xamanismo numa comunidade de pescadores (L'île enchantée : médecine et chamanisme dans un village de pêcheurs), Belém, Editora da Universidade, 1990.

3. B. Mindlin, Moqueca de maridos : mitos eróticos, Rio de Janeiro, Rosa dos Tempos, $2^{\mathrm{e}}$ éd., 1998 (Fricassée de maris, mythes érotiques amazoniens, Paris, Métailié, 2005) ; Mitos Indígenas, São Paulo, Ática, 2006 ; Carnets sauvages : chez les Surui du Rondônia, Paris, Métailié, 2008.

4. R. H. Maués, op. cit., p. 54.

5. Poisson typique de cette région. On lui attribue des pouvoirs magiques, tel celui de se transformer en un beau garçon pour séduire les filles. 
Quand la jeune fille écouta cette histoire, elle commença à se sentir mieux et après quelques semaines elle fut complètement rétablie. Selon Maués, ce qui a guéri la jeune fille, ce sont les paroles du chaman et les frères qui, par magie, auraient tué le boto. Dans ce cas, comme dans d'autres récits ${ }^{6}$, la pensée magique est présente dans les processus de guérison : le chaman est supposé avoir le pouvoir de communiquer avec les esprits responsables de guérir le malade et, au moment donné, de prononcer les mots porteurs de guérison.

D'après ce récit, ce que nous pouvons imaginer c'est qu'en effet, ce sont les effets des paroles du chaman qui ont fonctionné comme tiers dans la relation mère-fille où toute la question de la sexualité et la rivalité féminine entre la jeune fille et sa mère est présente, mais également la rivalité entre les frères et l'homme supposé procéder leur sœur. C'était comme si, dans sa position du tiers, les paroles du chaman avaient le même statut que celui de l'interprétation qui permet de symboliser le symptôme.

Pour la psychanalyse, les récits mythiques ont toujours leurs mots à dire à propos de l'histoire des constructions psychiques. C'est dans ce sens que la pensée magique occupe une place très importante dans la formation du psychisme. Selon l'hypothèse avancée par Freud dans un texte extrêmement spéculatif, Vue d'ensemble des névroses de transfert, la pensée magique serait à situer à un moment donné - forcément mythique - au long du chemin de l'évolution humaine (la phylogenèse), que chaque être humain doit refaire dans son histoire personnelle (l'ontogenèse). Cette étape, vécue dans la période prégénitale, donc avant le complexe d'CEdipe [avant Totem et tabou pour l'histoire de l'humanité], correspond à la récapitulation de la conception animiste du monde : « Le langage était pour lui [pour l'homme primitif, mais aussi pour l'enfant à cette période-là] de la magie, ses pensées lui semblaient être toute-puissantes. C'est le temps de la conception animiste du monde et de sa technique magique ${ }^{7}$. »

Cela fut alors dans ce temps premier que les mots acquirent leur «puissance magique », telle que celle de nommer les choses. Ce sentiment, que l'on appelle la toute-puissance infantile de la pensée magique, persiste tout au long de notre vie. L'enfant croit que le simple fait de prononcer une parole (parfois sacrée) engendrerait une action sur la réalité ayant le pouvoir de création. Dans cet âge des " caprices » infantiles, l'enfant ne comprend guère pourquoi ses demandes ne se réalisent point sur le champ. Ce ne sera pas sans « colère » qu'il devra se résigner au principe de réalité. Ce sera par le traitement psychique que peu à peu il va pouvoir entrer dans le principe de réalité.

6. B. Mindlin, Mitos Indígenas, op. cit.

7. S. Freud (1915), Vue d'ensemble des névroses de transfert, Paris, Gallimard, 1986, p. 37. 
La pensée magique, qui peuple les contes de fées ${ }^{8}$, aide $1^{\prime}$ enfant à grandir et crée à la fois un réservoir fantasmatique vers lequel nous nous retournons dans l'espoir d'y retrouver l'âge d'or : " Le charme de [notre] enfance, qu'un souvenir non impartial [nous] reflète comme un temps de bonheur sans trouble ${ }^{9}$. »

Dans les textes déjà cités ici, Freud aborde la nécessité impérieuse de l'être humain de se construire des théories, sorte de pensée magique, telles les théories sexuelles infantiles. Cette tendance irrépressible de l'être humain à rechercher des explications, l'épistémophilie, trouverait sa forme de pensée résiduelle dans une époque ancienne.

La position anthropologique originelle est que tout être humain arrive au monde au bout de neuf mois, non fini, donc dans la contrainte d'être pris en charge par un autre. C'est sur ce terrain de la prise en charge par un autre que son psychisme se dessinera. J. Laplanche, dans ses développements théoriques sur la " Théorie de la séduction généralisée ${ }^{10}$ », rappellera cette idée d'une situation anthropologique fondamentale qui caractérise l'être humain et fonde sa psyché. Conjointe à cette situation anthropologique fondamentale se trouve aussi la position dissymétrique dans laquelle se trouve l'enfant visà-vis de l'adulte qui lui donne les soins nécessaires. Position dissymétrique essentielle sur le plan de la sexualité qui vient inscrire à jamais la présence du sexuel dans tout mouvement psychique. Dont la pensée magique est un de ses avatars.

C'est à partir de cette situation entre un adulte qui a un inconscient marqué par le sexuel, constitué à partir des résidus de sa propre sexualité infantile, et un enfant qui n'a pas encore les «montages sexuels génétiques » pour comprendre et élaborer ce sexuel adulte que la rencontre s'installera. Cette situation de " séduction originaire ${ }^{11}$ » fonctionnera dans la mise en place de l'appareil psychique chez le petit enfant, conjointement au développement de sa sexualité.

Les questions que soulève cette vision d'une théorie de la séduction originaire sont nombreuses. Ce qui nous paraît très important, c'est qu'avec l'idée de dissymétrie sur le plan de la sexualité s'introduit la question du couple activité-passivité dans la dynamique psychique. Il ne s'agit pas seulement d'un adulte qui accompagne et qui porte les soins nécessaires à l'enfant, mais d'un adulte, la mère, qui introduit de la sexualité malgré elle.

Le geste de nourrir, par exemple, ne se réduit pas seulement à apporter de la nourriture. Dans ce geste il y a aussi un sein qui porte en lui une histoire

8. B. Bettelheim (1976), Psychanalyse des contes de fées, Paris, Hachette, 1998.

9. S. Freud (1939), L'homme Moïse et la religion monothéiste, Paris, Gallimard, 1986, p. 157.

10. J. Laplanche, Nouveaux fondements pour la psychanalyse, Paris, PUF, 1987.

11. Ibid. 
libidinale. Autrement dit, ce sein nourricier devient aussi érotique. Aux moments des soins donnés à l'enfant, il y a quelque chose qui s'implante et qui en même temps produit de l'excitation. Ce lien originaire du tout début de la vie reste donc contaminé par des messages chargés du sexuel adulte, constituant ce que J. Laplanche désigne par le terme, " messages énigmatiques ». Énigmatiques, puisque sexuels, énigmatiques, puisque l'enfant n'a pas encore accès à une compréhension de ce qui lui arrive. Ce terme " messages énigmatiques » prend toute son importance, parce qu'avec eux un autre mouvement vient s'ajouter. Cet autre mouvement est la nécessité de traduire ces messages pour les intégrer au psychisme qui est en train de se constituer.

Dans un premier temps, les messages, énigmatiques, sont implantés, sans être pour autant compris. C'est seulement dans un deuxième temps, qui se constitue dans des mouvements d'après-coups tout au long de l'évolution de l'enfant, que ces messages reviendront de l'intérieur pour trouver des traductions possibles. Les idéaux culturels seront donc les supports à fabriquer des formes de traductions pour donner sens aux mouvements internes et externes qui traversent l'enfant.

C'est dans ces zones encore de non-différenciation, où baigne le sentiment d'omnipotence, que les frontières psychiques se construiront. Les blessures narcissiques introduites à partir de la reconnaissance de l'altérité et du fait que le monde ne tourne pas comme l'enfant voulait seront décisives pour la construction de l'organisation psychique de l'enfant, mais ainsi retrouveront une forme d'expression dans les constructions mythiques.

Ce que nous pouvons dire comme étant universel, c'est cette position primitive où la condition de prématurité de l'enfant le met dans une situation de dépendance vis-à-vis de l'autre, et cela dans n'importe quelle culture. Ce qui va se différencier d'une culture à une autre seront les supports à traduire que chaque culture offre, c'est-à-dire le système de valeur, les idéaux dans lesquels le tout-petit se trouve submergé dès sa naissance.

A. Green, dans son texte Idées directrices pour une psychanalyse contemporaine ${ }^{12}$ revient sur la réflexion de cette situation primitive propre à tout être humain en mettant l'accent sur l'expérience de négativité présente dans la rencontre avec l'objet source de satisfaction. Le nourrisson, face à l'expérience d'absence de l'objet source de satisfaction, aura comme solution psychique son hallucination pendant un certain temps. Il fera à partir de cette première expérience d'hallucination, des constructions psychiques appuyées sur sa culture.

12. A. Green, Idées directrices pour une psychanalyse contemporaine, Paris, PUF, 2002. 
Il sera donc possible de penser à une potentialité hallucinatoire, avec des destins différents selon la culture dans laquelle elle se trouve inscrite. Permettant ainsi des constructions individuelles et collectives où la réalité psychique s'opposera ou tentera de s'opposer ou composera avec la réalité propre à chaque contexte culturel.

Un des collègues travaillant dans un hôpital à Belém, la capitale de l'État du Pará ${ }^{13}$, nous a rapporté le cas suivant. Il s'agissait d'une femme d'origine indienne venue à l'hôpital à cause d'une toux qui ne la lâchait pas. Les examens médicaux ont révélé un problème pulmonaire et il lui a été fermement conseillé d'arrêter de fumer immédiatement. Quelques mois après, lors d'une consultation suivante, elle a dit qu'elle ne pouvait pas arrêter de fumer et a refusé de dire la raison qui l'empêchait de le faire. Sur l'insistance des médecins, soutenus par son mari et ses enfants, elle est finalement tombée d'accord pour parler au chaman, le guérisseur du village d'où elle venait. Suite à cette rencontre avec le chaman, elle a accepté d'arrêter de fumer. La raison qui l'empêchait d'arrêter le tabac, c'était la peur qu'elle éprouvait visà-vis de Matita Perê.

Matita Perê est une entité mythique. Il s'agissait d'une jeune fille nommée Matita Pereira (ou Matita Perê) qui était de son vivant une femme gaie et heureuse, dont le passe-temps préféré était celui de fumer. Néanmoins, elle avait un problème : son mari, très coléreux et jaloux. Un jour, quand Matita était enceinte, son mari rentra ivre et en colère. Les deux se bagarrèrent terriblement et l'homme finit par tuer sa femme. Mais comme Matita était protégée par des forces surnaturelles, elle reçut un don : son esprit gagna la capacité de se transformer en femme pendant la journée et en chouette la nuit. Comme elle aimait fumer, le jour, elle frappe aux maisons en demandant du tabac. Si quelqu'un refuse de lui en donner, Matita revient le soir pour mettre le fœtus de son enfant mort sur le seuil de la maison de cette personne. Par ailleurs, Matita a aussi la capacité de percevoir quand quelqu'un va mourir dans une famille, et de ce fait, le soir, elle se transforme en une petite chouette et rôde autour de la maison. Pour cette femme venue à l'hôpital, selon la pensée magique, arrêter le tabac, c'était prendre le risque de ne pas avoir de tabac à offrir à Matita Perê et donc être soumise à la présence de la mort.

Pour revenir à notre question du début de ce texte - le rapprochement entre deux mouvements de pensées (psychanalytique et anthropologique

13. Cet hôpital, situé dans sa capitale, accueille surtout une population pauvre venue des quatre coins de l'État. Nombre d'entre eux viennent du fin fond de la forêt amazonienne et ont parfois besoin de plusieurs jours de déplacement pour arriver jusqu'à l'hôpital. Ces hommes et ces femmes, bien qu'acculturés, maintiennent néanmoins les mœurs et traditions, bref, l'imaginaire, propres à leur société. (C. Lindenmeyer, P. R. Ceccarelli, « Traumatisme et sexualité », Recherches en psychanalyse, 5, Paris, 2006, p. 111-118.) 
par rapport à la pensée magique) à la fois opposées et si proches -, il nous semble qu'une première ébauche de réponse serait dans les rapports profonds présents entre ces deux mouvements de pensée. En ce qui concerne leur fonction première : ils sont des tentatives de faire face à la détresse (Hilflosigkeit) originaire. Au tout début de la vie, le nouveau-né est incapable d'affronter les exigences pulsionnelles phylogénétiquement héritées ${ }^{14}$, en raison de l'absence d'un appareil psychique. Pour faire face à ce que l'on peut appeler à juste titre la « détresse psychique », des investissements libidinaux (identifications) sont faits pour donner des représentations aux pulsions, ce qui soutient le Moi en constitution. Parmi les identifications constitutives du Moi, il y a des associations syntagmatiques - y compris les pensées magiques - utilisés et par la culture et par l'individu pour « lire le monde ».

L'histoire libidinale de l'humanité nous montre que les dispositifs que nous créons pour nous réconforter ne sont jamais suffisants. Aucune organisation psychosociale, croyance religieuse, scientifique, aucune forme de lien social nous protège contre la position de détresse originaire ${ }^{15}$. Les constructions mythiques proposées par des «visions du monde » (Weltanschauung) que nous avons utilisées tout au long du processus évolutif - animiste, religieuse ou scientifique - sont toujours fragmentaires et susceptibles de se transformer en un système de croyance des masses ${ }^{16}$. Tous les discours puisent aux mêmes sources, les croyances des enfants - c'est-à-dire mythiques - dont les origines sont à chercher dans les théories sexuelles infantiles. Les discours sur les destins du pathos, des passions qui animent l'appareil de l'âme (seelischer Apparat), ne sont que des artefacts culturels créés pour essayer de comprendre et de traiter l'altérité interne : les manifestations de l'inconscient, preuve irréfutable que nous ne sommes pas maîtres dans notre propre la maison. En étant l'inconscient sexuel, ses productions sont souvent ressenties par le sujet et la culture comme de l'ordre de l'étrangeté (unheimlich), parfois une chose à éradiquer. Freud ne cache pas sa déception quant à l'inexorabilité de la détresse dans ses "Considérations actuelles sur la guerre et sur la mort ». Ce travail, un véritable « épanchement du cœur », annonce des changements dans ses positions théoriques, dont l'expression ultime est faite, non sans quelque réserve de la part du Freud lui-même, en 1920 avec l'introduction de la pulsion de mort.

14. S. Freud, Vue d'ensemble des névroses de transfert, op. cit.

15. P. R. Ceccarelli, « Laço social : uma ilusão frente ao desamparo » («Lien social : une illusion face à la désaide »), Reverso, Revista do Círculo Psicanalítico de Minas Gerais, ano XXXI, 58, p. 33-41, 2009.

16. S. Freud (1915), «Considérations actuelles sur la guerre et sur la mort», dans Essais de psychanalyse, Paris, Payot, 1987. 
Freud en parlant du primitif ou du sauvage dans Totem et tabou nous destituait d'une pensée totalitaire selon laquelle les névrosés ou l'homme civilisé se trouveraient dans une position de supériorité vis-à-vis duprimitif.

En plaçant les choses ainsi, Freud rappelle la blessure narcissique originaire de se sentir supérieur à l'autre, reconnu comme sauvage. "Afin de parachever cette satisfaction [narcissique], chaque civilisation se compare aux autres cultures, qui se sont consacrées à d'autres tâches et se sontérigées d'autres idéaux. Grâce à ces différences, chaque civilisation s'arroge le droit de mépriser les autres. C'est ainsi que les idéaux culturels deviennent une cause de discorde et d'inimitié, entre groupes culturels différents, ainsi qu'on peut clairement le voir entre nations ${ }^{17}$. "

Dans notre travail de réflexion avec d'autres collègues qui travaillent avec les populations indiennes, cette blessure rappelée par Freud, mais parfois oubliée, réapparaît avec force. Toute tendance à caractériser ses constructions culturelles dit plus sur «nous » que sur « eux ».

Dans ce travail de construction mythique de ces populations, leurs constructions psychiques, qui prennent leur source dans la pensée omnipotente et magique de l'enfant, paraissent garder leur puissance intacte. La confrontation avec ces constructions mythiques est parfois source de résistances multiples, parfois même source de visions normatives de ces populations.

Cette vision normative qui naît de la confrontation de la différence avec l'autre, différence qui prend aussi sa source dans ce vécu originaire omnipotent, peut parfois nous jouer des tours. Et si ce qui vient donner forme à l'étranger se trouvait à l'intérieur de nous-même ? Freud, dans son texte "L'inquiétante étrangeté ${ }^{18}$ », nous rappelle - et « enfonce le clou »-que ce que nous prenons pour étranger est en définitif le plus familier. À l'entrée de son compartiment de train, Freud est confronté à la vision du visage d'un homme âgé ; surpris quelques minutes, il se rend compte que cet homme, qu'il ne reconnaît pas, est en fait lui-même.

\section{Résumé}

Les auteurs développent l'idée que la pensée magique trouvera aussi sa forme d'expression dans d'autres cultures. À partir des exemples rencontrés par des anthropologues, les auteurs abordent ce « reliquat de la mentalité primitive » qui subsiste toujours sous une forme latente dans le psychisme de tout un chacun. Les auteurs développent leurs réflexions appuyées sur l'idée que la pensée magique constituerait une tentative d'échapper aux angoisses et aux conflits et du monde externe et

17. S. Freud (1927), L'avenir d'une illusion, Paris, PUF, 4 e éd., 1973.

18. S. Freud (1919), L'inquiétante étrangeté et autres essais, Paris, Gallimard, 1991. 
du monde interne ; comme si l'acte de penser pouvait contrôler, expliquer et même changer la réalité.

Mots-clés

Pensée magique, désaide, inquiétante étrangeté, développement psychique.

AVATARS OF MAGIC THOUGHTS

Summary

The authors develop the idea according to which expressions of magical thinking can also be found in other cultures. From examples described by anthropologists the authors try to identify this " primitive residual mentality » that still exists in latent form in the psyche of each human being. Magical thinking is an attempt to escape anxiety and conflict from both external and internal world, as if the act of thinking could control, explain, and even change reality.

Keywords

Magical thinking, helplessness, uncanny, psychic development. 